\title{
Novel one-pot synthesis of luminescent neutral rhenium-based molecular rectangles
}

\author{
Bala. Manimaran, ${ }^{a}$ T. Rajendran, ${ }^{a} \dagger$ Yi-Long Lu, ${ }^{a}$ Gene-Hsiang Lee, ${ }^{b}$ Shie-Ming Peng ${ }^{b}$ \\ and Kuang-Lieh Lu*a \\ ${ }^{a}$ Institute of Chemistry, Academia Sinica, Taipei 115, Taiwan, ROC. \\ E-mail: lu@chem.sinica.edu.tw \\ ${ }^{b}$ Department of Chemistry, National Taiwan University, Taipei 107, Taiwan, ROC
}

Received 30th January 2001, Accepted 31st January 2001

First published as an Advance Article on the web 14th February 2001

\begin{abstract}
The one-pot synthesis of soluble, neutral, luminescent rectangular supramolecules in high yields by solvothermal methods and fine tuning of the molecular architecture in one dimension from $\sim 7 \AA$ to a nanometer scale, $\sim 21 \AA$, have been achieved.
\end{abstract}

Metallomacromolecules possessing interesting inclusion, light harvesting and molecular recognition properties form a hot subject of current research in chemistry and materials science. ${ }^{1,2}$ In recent years many workers have reported the synthesis, characterization and applications of luminescent molecular squares based on $\operatorname{Re}(\mathrm{CO})_{3}$ corners with pyridine-based ligands as bridges. ${ }^{3,4}$ Compared to a molecular square a rectangle may have advantages in host-guest chemistry and in molecular recognition leading to altered chemical selectivity and/or enhanced binding of planar aromatic guest species. However only a few reports appeared on $\operatorname{Re}(\mathrm{I})$ containing rectangles from the schools of Hupp 5 and Sullivan ${ }^{6}$ and from this laboratory. Though the presence of lengthy alkyl chains may expand the applications of macromolecules, the synthesis of $\operatorname{Re}(\mathrm{I})$ supramolecules bearing lengthy alkoxy bridges are hitherto unknown. Keeping the above aspects in mind, work has been initiated in this laboratory on the synthesis of neutral molecular rectangles from $\operatorname{Re}_{2}(\mathrm{CO})_{10}$. A facile solvothermal approach for the synthesis of metallomacromolecules possessing long hydrophobic chains is reported in this communication. This strategy leads to the high-yield formation of the self-assembled products.

When $\operatorname{Re}_{2}(C O)_{10}$ is treated with nitrogen-containing ligands, pyrazine (pz), trans-1,2-bis(4-pyridyl)ethylene (bpe) and 1,4-bis[2-(4-pyridyl)ethenyl]benzene (bpeb) in the presence of higher aliphatic alcohols, using solvothermal methods, the alkoxy bridged molecular rectangles $\left[\left\{(\mathrm{CO})_{3} \operatorname{Re}(\mu-\mathrm{OR})_{2} \operatorname{Re}(\mathrm{CO})_{3}\right\}_{2}{ }^{-}\right.$ $\left.(\mu-\mathrm{L})_{2}\right](\mathbf{1}-\mathbf{3})$ are obtained $\$$ (Scheme 1$)$. Rectangles 1-3 have been spectroscopically characterized. The more hydrophobic nature of the rectangles containing an octyl group compared to those carrying a butyl group enhanced the solubility in less polar solvents.

X-Ray quality crystals were obtained by solvothermal means and an X-ray diffraction study was carried out for 3a.§ The ORTEP diagram of 3a (Fig. 1) revealed a rectangular architecture, where two planar bpeb ligands are coordinated to four rhenium atoms, which are connected by four butoxy bridges thereby forming a molecular rectangle. Each rhenium atom occupies the corner of the rectangle and is bonded to one nitrogen atom of the pyridyl group of the bpeb ligand, two butoxy groups and three carbonyl groups. In each bridging ligand (bpeb), the two pyridyl groups and the central aromatic ring are oriented in the same plane.

$\dagger$ Permanent address: Department of Chemistry, Vivekananda College, Tiruvedakan West, 625 217, Madurai, India.

DOI: $10.1039 / \mathrm{b} 101020$ o

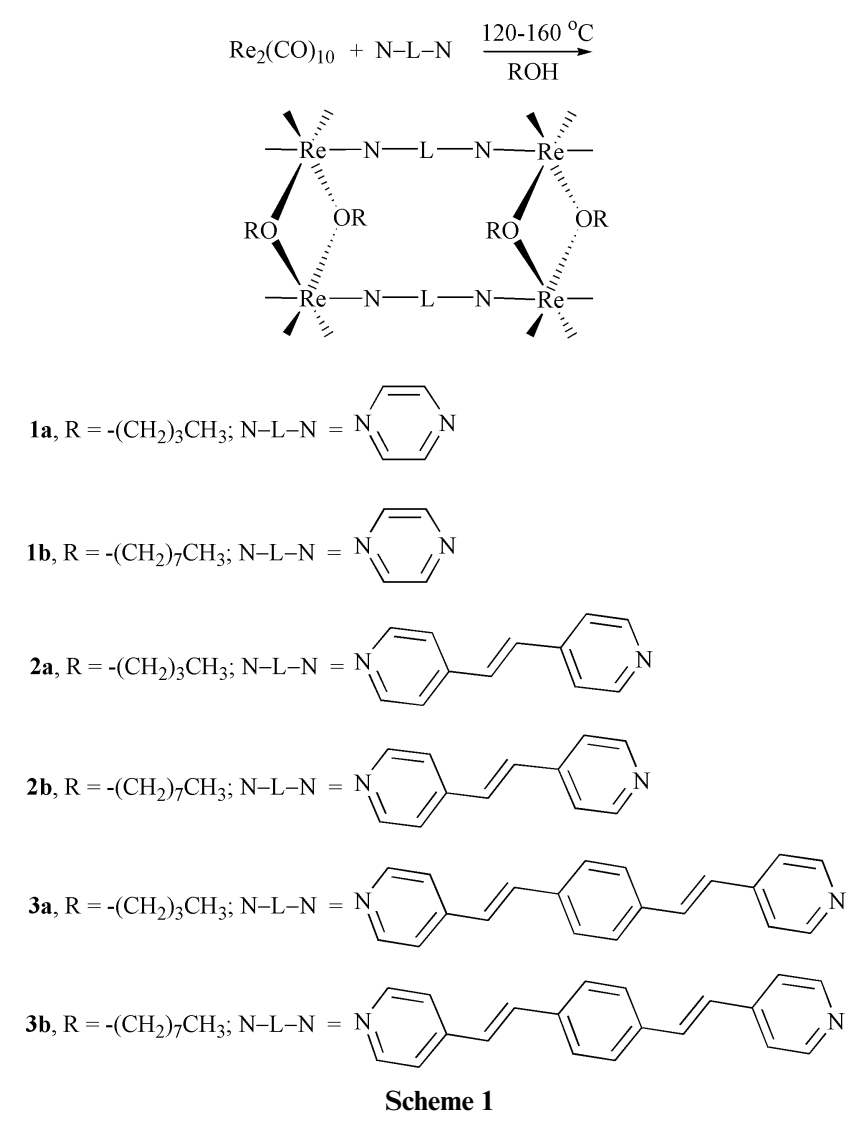

The IR spectra of $\mathbf{1}-\mathbf{3}$ show a gradual shift in the carbonyl stretching frequencies, when the ligand is changed from bpeb (bpe) to pz. ${ }^{8,9}$ This indicates that among the three ligands used, the coordinating ability of bpeb and bpe is comparable to but stronger than pz. Electronic absorption spectral studies of 1-3 revealed the existence of two types of bands, one in the UV and the other in the visible region. In a broad way we can assign the higher energy features to the ligand $\left(\pi-\pi^{*}\right)$ based transition, and the lower energy absorption to the metal-to-ligand (bridging) charge transfer (MLCT) transition. ${ }^{10}$ The higher energy absorption $\left(\pi-\pi^{*}\right)$ is always intense, about four fold stronger than the MLCT transition. A red shift of $61 \mathrm{~nm}$ (from $398 \mathrm{~nm}$ to $459 \mathrm{~nm}$ ) is seen in the MLCT absorption maxima of these compounds on changing the ligand from bpe to pz. The observation that the more easily reducible ligands yield complexes exhibiting lower energy absorption, is in agreement with the results of Wrighton and others. ${ }^{10}$ With the molecular squares, a change of ligand from bpe to pz shifts the lower energy $\lambda_{\max }$ from $358 \mathrm{~nm}$ to $396 \mathrm{~nm} .{ }^{11}$ Thus the alkoxy bridges facilitate

J. Chem. Soc., Dalton Trans., 2001, 515-517

515 


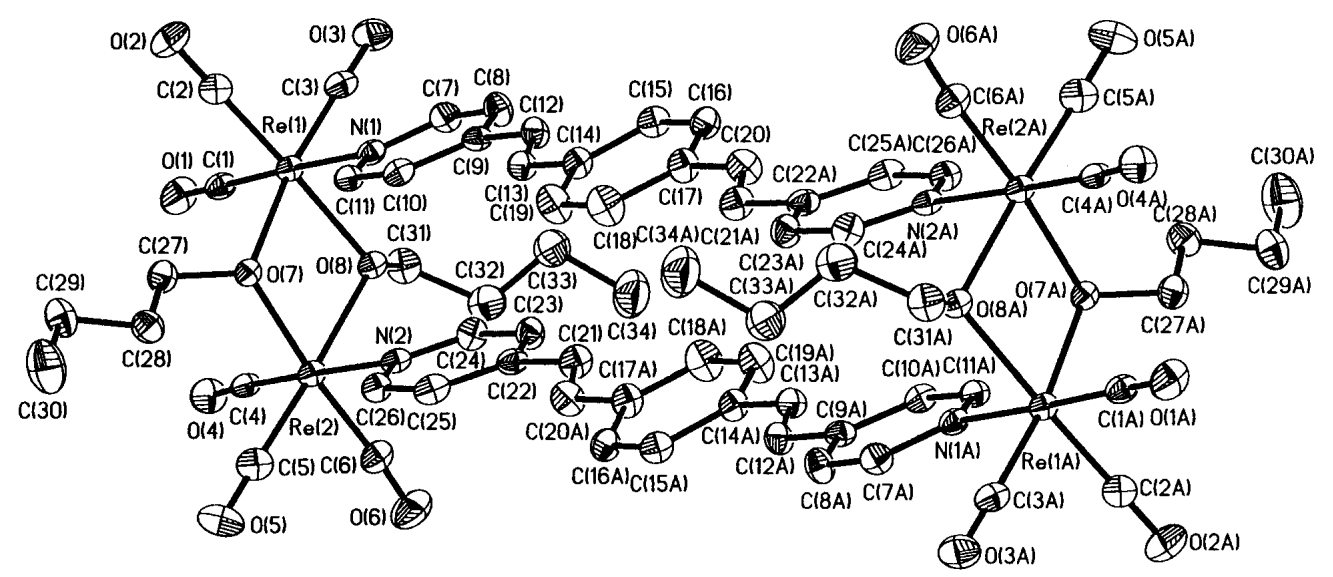

Fig. 1 ORTEP ${ }^{16}$ diagram of $\left[\left\{(\mathrm{CO})_{3} \operatorname{Re}\left(\mu-\mathrm{OC}_{4} \mathrm{H}_{9}\right)_{2} \operatorname{Re}(\mathrm{CO})_{3}\right\}_{2}(\mu \text {-bpeb })_{2}\right](3 \mathrm{a})$. Selected bond distances $(\AA)$ and angles $\left({ }^{\circ}\right): \operatorname{Re}(1)-\mathrm{N}(1) 2.219(4)$, $\operatorname{Re}(1)-\mathrm{O}(7) 2.154(3), \operatorname{Re}(1)-\mathrm{O}(8) 2.161(3), \operatorname{Re}(1)-\mathrm{C}(1)$ 1.923(5), $\operatorname{Re}(1)-\mathrm{C}(2) 1.905(6), \operatorname{Re}(1)-\mathrm{C}(3)$ 1.912(6), O(7)-C(27) 1.447(6), O(8)-C(31) 1.451(6); $\mathrm{O}(7)-\operatorname{Re}(1)-\mathrm{N}(1)$ 82.36(14), O(8)-Re(1)-N(1) 83.29(13), Re(1)-O(7)-Re(2) 104.06(14), $\operatorname{Re}(1)-\mathrm{O}(8)-\operatorname{Re}(2) 103.49(14), \mathrm{O}(7)-\operatorname{Re}(1)-\mathrm{O}(8)$ 74.68(13), $\mathrm{O}(7)-\operatorname{Re}(2)-\mathrm{O}(8)$ 74.53(13), C(27)-O(7)-Re(1) 118.1(3), C(27)-O(7)-Re(2) 123.3(3), C(31)-O(8)-Re(1) 117.0(3), C(31)-O(8)-Re(2) 118.2(3).

$\mathrm{Re} \longrightarrow \mathrm{L}$ charge transfer. It is interesting to note that the (MLCT) bands of $\mathbf{1 a}$ and $\mathbf{1 b}$ are highly sensitive to a change in the polarity of the solvent and exhibit solvatochromism, having a shift in $\lambda_{\max }$ to the tune of $100 \mathrm{~nm}$ when the solvent is varied from DMSO to $\mathrm{CCl}_{4}$. Excitation of the complexes near the MLCT absorption wavelength results in moderate emission in the range 440 to $490 \mathrm{~nm}$ in $\mathrm{CH}_{3} \mathrm{CN}$ and $\mathrm{CH}_{2} \mathrm{Cl}_{2}$. However, the free ligands also show emission in the above region. Since both the free ligands and rectangles exhibit emissions around the same region, the observed emission for the complexes may therefore originate from the MLCT band involving primarily the bridging ligands. The excited state lifetimes of these rectangles have been measured and are in the range of 10 to 15 ns at room temperature.

The measured cyclic voltammetric data show that each of the complexes studied here displays two or three reduction waves and one to four oxidation waves. The first reduction wave in the range -0.73 to $-1.28 \mathrm{~V}$ can be assigned to the first reduction of the bridging ligand, $\mathrm{L} / \mathrm{L}^{-12-15}$ The second reduction observed with the complexes containing bpe and bpeb at $-1.26 \mathrm{~V}$ and $-1.28 \mathrm{~V}$ may be assigned to the second reduction of the ligand, $\mathrm{L}^{-} / \mathrm{L}^{2-}$. The reduction wave observed in the range -1.35 to $-1.50 \mathrm{~V}$ in the case of complexes containing bpe and pz can be assigned to the reduction of the metal, $\mathrm{Re}^{+} / \mathrm{Re}^{0}{ }^{13,15}$ The oxidation peaks observed in the range $1.00-1.65 \mathrm{~V}$ may be attributed to the oxidation of the four metal centers present in the rectangle. As the alkoxy bridge remains as a good electron donor in the present system it is not able to show any redox reaction in the potential range -2.00 to $2.00 \mathrm{~V}$.

In conclusion, we have demonstrated that a novel class of molecular rectangles bearing octahedral Re centers can be prepared by a one-pot synthesis in high yield by solvothermal methods. The solubility of these neutral rectangles can be tuned by tailoring the length of the alkyl chain of the alkoxy group. The luminescence and the solvatochromic properties make these molecular rectangles interesting species. Further work is in progress to tune the luminescence properties of these molecular rectangles in order to expand their applications.

We thank Academia Sinica and the National Science Council of Taiwan for financial support. We are also grateful to Professor S. Rajagopal for valuable discussions.

\section{Notes and references}

$\$$ General synthetic procedure for 1-3: In a typical preparation, a suspension containing a mixture of $\mathrm{Re}_{2}(\mathrm{CO})_{10}$ and the appropriate $\mathrm{N}$-ligand in $10 \mathrm{ml}$ aliphatic alcohol in a $30 \mathrm{ml}$ Teflon flask was placed in an oven maintained at $120-160^{\circ} \mathrm{C}$ for $48 \mathrm{~h}$ and then cooled to $25^{\circ} \mathrm{C}$ The crystals were separated by filtration and the solvent was removed from the filtrate under vacuum, the residue was then redissolved in the minimum quantity of $\mathrm{CH}_{2} \mathrm{Cl}_{2}$ and passed through a short silica gel column to obtain the pure product. Yield: 1a, $71 \% ; \mathbf{1 b}, 73 \% ; \mathbf{2 a}, 76 \%$; 2b, $82 \%$; 3a, $78 \%$; 3b, $84 \%$.
Compound 1a: IR $\left(\mathrm{CH}_{2} \mathrm{Cl}_{2}\right)$ : v $v_{\mathrm{CO}} 2024$ (s), 2017 (sh, m), 1925 (m), 1903 (vs) cm ${ }^{-1} ;{ }^{1} \mathrm{H}$ NMR [ $\left.300 \mathrm{MHz},\left(\mathrm{CD}_{3}\right)_{2} \mathrm{CO}\right]: \delta 8.83\left(\mathrm{~s}, 8 \mathrm{H}, \mathrm{H}^{2}\right), 4.44$ $(\mathrm{m}, 8 \mathrm{H}), 2.17(\mathrm{~m}, 8 \mathrm{H}), 1.48(\mathrm{~m}, 8 \mathrm{H}), 1.07\left(\mathrm{t},{ }^{3} \mathrm{~J} 7.4 \mathrm{~Hz}, 12 \mathrm{H}\right) ;{ }^{13} \mathrm{C}$ NMR [75 MHz, $\left.\left(\mathrm{CD}_{3}\right)_{2} \mathrm{CO}\right]: \delta$ 199.0, $198.2(1: 2, \mathrm{CO}), 150.1\left(\mathrm{C}^{2}\right.$, pyrazine), $82.9\left(\mathrm{CH}_{2}\right), 36.5\left(\mathrm{CH}_{2}\right), 19.0\left(\mathrm{CH}_{2}\right), 14.5\left(\mathrm{CH}_{3}\right) ; \mathrm{UV}-\mathrm{VIS}$ $\left(\mathrm{CH}_{3} \mathrm{CN}\right): \lambda_{\text {max }}$ (nm) 452 (MLCT), 255, 261, 268 (LIG). Anal. for $\mathrm{C}_{36} \mathrm{H}_{44} \mathrm{~N}_{4} \mathrm{O}_{16} \mathrm{Re}_{4}$, found (calc.): C, 28.09 (28.19); H, 2.85 (2.89); N, 3.73 $(3.65) \%$. Mass (FAB, $\left.{ }^{187} \mathrm{Re}\right): \mathrm{m} / \mathrm{z} 1536\left(\mathrm{M}^{+}\right)$.

Compound 1b: IR $\left(\mathrm{CH}_{2} \mathrm{Cl}_{2}\right)$ : $v_{\mathrm{CO}} 2024$ (s), 2018 (sh, m), 1924 (m), 1903 (vs) cm ${ }^{-1}$; ${ }^{1} \mathrm{H}$ NMR [300 MHz, $\left.\left(\mathrm{CD}_{3}\right)_{2} \mathrm{CO}\right]: \delta 8.83\left(\mathrm{~s}, 8 \mathrm{H}, \mathrm{H}^{2}\right)$, $4.42(\mathrm{~m}, 8 \mathrm{H}), 2.20(\mathrm{~m}, 8 \mathrm{H}), 1.46(\mathrm{~m}, 24 \mathrm{H}), 1.35(\mathrm{~m}, 16 \mathrm{H}), 0.91(\mathrm{t}$, $\left.{ }^{3} J 6.9 \mathrm{~Hz}, 12 \mathrm{H}\right) ;{ }^{13} \mathrm{C}$ NMR $\left[75 \mathrm{MHz},\left(\mathrm{CD}_{3}\right)_{2} \mathrm{CO}\right]: \delta 198.7,197.1(1: 2$, $\mathrm{CO}), 150.0\left(\mathrm{C}^{2}\right.$, pyrazine), 83.1 $\left(\mathrm{CH}_{2}\right), 34.2\left(\mathrm{CH}_{2}\right), 32.5\left(\mathrm{CH}_{2}\right), 30.2$ $\left(\mathrm{CH}_{2}\right), 29.9\left(\mathrm{CH}_{2}\right), 25.7\left(\mathrm{CH}_{2}\right), 23.3\left(\mathrm{CH}_{2}\right), 14.3\left(\mathrm{CH}_{3}\right) ;$ UV-VIS $\left(\mathrm{CH}_{3} \mathrm{CN}\right): \lambda_{\max }(\mathrm{nm}) 459$ (MLCT), 255, 261 (LIG); emission: $\lambda_{\max }(\mathrm{nm})$ 470. Anal. for $\mathrm{C}_{52} \mathrm{H}_{76} \mathrm{~N}_{4} \mathrm{O}_{16} \mathrm{Re}_{4}$, found (calc.): C, 35.97 (35.53); $\mathrm{H}, 4.30$ (4.36); N, $3.08(3.18)^{\%} \%$. Mass (FAB, $\left.{ }^{187} \mathrm{Re}\right): m / z 1760\left(\mathrm{M}^{+}\right)$.

Compound 2a: IR $\left(\mathrm{CH}_{2} \mathrm{Cl}_{2}\right): v_{\mathrm{CO}} 2020(\mathrm{~s}), 2008(\mathrm{~m}), 1909(\mathrm{~m}), 1888$ (vs) $\mathrm{cm}^{-1} ;{ }^{1} \mathrm{H}$ NMR [300 MHz, $\left(\mathrm{CD}_{3}\right)_{2} \mathrm{CO}$ ]: $\delta 8.46\left(\mathrm{~d},{ }^{3} J 5.3 \mathrm{~Hz}, 8 \mathrm{H}\right.$, $\left.\mathrm{H}^{3}\right), 7.63$ (s, $4 \mathrm{H}$, vinyl), $7.59\left(\mathrm{~d},{ }^{3} \mathrm{~J} 5.3 \mathrm{~Hz}, 8 \mathrm{H}, \mathrm{H}^{2}\right), 4.41(\mathrm{~m}, 8 \mathrm{H}), 2.13$ $(\mathrm{m}, 8 \mathrm{H}), 1.49(\mathrm{~m}, 8 \mathrm{H}), 1.08\left(\mathrm{t},{ }^{3} \mathrm{~J} 7.4 \mathrm{~Hz}, 12 \mathrm{H}\right) ;{ }^{13} \mathrm{C}$ NMR [125 MHz; $\left.\left(\mathrm{CD}_{3}\right)_{2} \mathrm{CO}\right]: \delta$ 198.9, $198.5(1: 2, \mathrm{CO}), 152.8\left(\mathrm{C}^{3}\right), 146.4\left(\mathrm{C}^{1}\right), 132.6$ (vinyl), $124.0\left(\mathrm{C}^{2}\right), 82.5\left(\mathrm{CH}_{2}\right), 36.5\left(\mathrm{CH}_{2}\right), 19.1\left(\mathrm{CH}_{2}\right), 14.5\left(\mathrm{CH}_{3}\right)$; UVVIS ( $\mathrm{CH}_{3} \mathrm{CN}$ ): $\lambda$ (nm) 398 (MLCT), 289 (LIG); emission: $\lambda$ (nm) 440, 454. Anal. for $\mathrm{C}_{52} \mathrm{H}_{56} \mathrm{~N}_{4} \mathrm{O}_{16} \mathrm{Re}_{4}$, found (calc.): $\mathrm{C}, 35.98$ (35.94); $\mathrm{H}, 3.27$ (3.25); N, $3.33(3.22) \%$. Mass (FAB, $\left.{ }^{187} \mathrm{Re}\right): \mathrm{m} / \mathrm{z} 1740\left(\mathrm{M}^{+}\right)$.

Compound 2b: IR $\left(\mathrm{CH}_{2} \mathrm{Cl}_{2}\right): v_{\mathrm{Co}} 2020(\mathrm{~s}), 2008(\mathrm{~m}), 1909(\mathrm{~m}), 1886$ (vs) $\mathrm{cm}^{-1}$; ${ }^{1} \mathrm{H}$ NMR [ $300 \mathrm{MHz},\left(\mathrm{CD}_{3}\right)_{2} \mathrm{CO}$ ]: $\delta 8.46\left(\mathrm{~d},{ }^{3} J 6.6 \mathrm{~Hz}, 8 \mathrm{H}\right.$, $\left.\mathrm{H}^{3}\right), 7.62\left[\mathrm{~s}, 4 \mathrm{H}\right.$, (vinyl)], $7.57\left(\mathrm{~d},{ }^{3} J 6.6 \mathrm{~Hz}, 8 \mathrm{H}, \mathrm{H}^{2}\right), 4.39(\mathrm{~m}, 8 \mathrm{H}), 2.16$ $(\mathrm{m}, 8 \mathrm{H}), 1.47(\mathrm{~m}, 24 \mathrm{H}), 1.36(\mathrm{~m}, 16 \mathrm{H}), 0.92\left(\mathrm{t},{ }^{3} \mathrm{~J} 6.8 \mathrm{~Hz}, 12 \mathrm{H}\right) ;{ }^{13} \mathrm{C}$ NMR [75 MHz; $\left(\mathrm{CD}_{3}\right)_{2} \mathrm{CO}$ ]: $\delta$ 199.0, $198.6(1: 2, \mathrm{CO}), 152.1\left(\mathrm{C}^{3}\right), 146.4$ $\left(\mathrm{C}^{1}\right), 132.7$ (vinyl), $124.1\left(\mathrm{C}^{3}\right), 82.8\left(\mathrm{CH}_{2}\right), 34.4\left(\mathrm{CH}_{2}\right), 32.6\left(\mathrm{CH}_{2}\right), 30.4$ $\left(\mathrm{CH}_{2}\right), 30.0\left(\mathrm{CH}_{2}\right), 26.0\left(\mathrm{CH}_{2}\right), 23.3\left(\mathrm{CH}_{2}\right), 14.4\left(\mathrm{CH}_{3}\right) ; \mathrm{UV}-\mathrm{VIS}$ $\left(\mathrm{CH}_{3} \mathrm{CN}\right): \lambda_{\max }(\mathrm{nm}) 398$ (MLCT), 289 (LIG); emission: $\lambda_{\max }(\mathrm{nm}) 460$. Anal. for $\mathrm{C}_{68} \mathrm{H}_{88} \mathrm{~N}_{4} \mathrm{O}_{16} \mathrm{Re}_{4}$, found (calc.): C, 41.58 (41.62); H, 4.47 (4.52); $\mathrm{N}, 2.85(2.86) \%$. Mass (FAB, $\left.{ }^{187} \mathrm{Re}\right): m / z 1964\left(\mathrm{M}^{+}\right)$.

Compound 3a: IR $\left(\mathrm{CH}_{2} \mathrm{Cl}_{2}\right): v_{\mathrm{CO}} 2019(\mathrm{~s}), 2005(\mathrm{~m}), 1905(\mathrm{~m}), 1884$ (vs) $\mathrm{cm}^{-1} ;{ }^{1} \mathrm{H}$ NMR [300 MHz, $\left.\left(\mathrm{CD}_{3}\right)_{2} \mathrm{CO}\right]: \delta 8.36\left[\mathrm{~d},{ }^{3} J 6.5 \mathrm{~Hz}, 8 \mathrm{H}\right.$, (pyridyl) $\left.\mathrm{H}^{3}\right], 7.54\left[\mathrm{~d},{ }^{3} J 16.4 \mathrm{~Hz}, 4 \mathrm{H}\right.$, (vinyl) $\left.\mathrm{H}^{2}\right], 7.47\left[\mathrm{~d},{ }^{3} J 6.5 \mathrm{~Hz}, 8 \mathrm{H}\right.$, (pyridyl) $\mathrm{H}^{2}$ ] 7.38 [s, $8 \mathrm{H}$ (phenyl)], 7.18 [d, ${ }^{3} J 16.4 \mathrm{~Hz}, 4 \mathrm{H}$ (vinyl) $\mathrm{H}^{1}$ ], $4.40(\mathrm{~m}, 8 \mathrm{H}), 2.13(\mathrm{~m}, 8 \mathrm{H}), 1.49(\mathrm{~m}, 8 \mathrm{H}), 1.08\left(\mathrm{t},{ }^{3} \mathrm{~J} 7.3 \mathrm{~Hz}, 12 \mathrm{H}\right)$; UV-VIS $\left(\mathrm{CH}_{3} \mathrm{CN}\right.$ ): $\lambda_{\max }$ (nm) 423 (MLCT), 269, 364 (LIG); emission: $\lambda_{\max }(\mathrm{nm})$ 474. Anal. for $\mathrm{C}_{68} \mathrm{H}_{68} \mathrm{~N}_{4} \mathrm{O}_{16} \mathrm{Re}_{4}$, found (calc.): $\mathrm{C}, 42.37$ (42.05); H, 3.42 (3.53); N, 2.92 (2.88)\%. Mass (FAB, ${ }^{187} \mathrm{Re}$ ): $\mathrm{m} / \mathrm{z} 1944$ $\left(\mathrm{M}^{+}\right)$.

Compound 3b: IR $\left(\mathrm{CH}_{2} \mathrm{Cl}_{2}\right)$ : $v_{\mathrm{CO}} 2020$ (s), 2006 (m), 1906 (m), 1883 (vs) $\mathrm{cm}^{-1},{ }^{1} \mathrm{H}$ NMR [ $\left.300 \mathrm{MHz},\left(\mathrm{CD}_{3}\right)_{2} \mathrm{CO}\right]: \delta 8.36\left[\mathrm{~d},{ }^{3} J 6.6 \mathrm{~Hz}, 8 \mathrm{H}\right.$, (pyridyl) $\mathrm{H}^{3}$ ] $7.53\left[\mathrm{~d},{ }^{3} J 16.4 \mathrm{~Hz}, 4 \mathrm{H}\right.$, (vinyl) $\mathrm{H}^{2}$ ], $7.46\left[\mathrm{~d},{ }^{3} J 6.6 \mathrm{~Hz}, 8 \mathrm{H}\right.$, (pyridyl) $\mathrm{H}^{2}$ ], 7.38 [s, $8 \mathrm{H}$, (phenyl)], 7.18 [d, ${ }^{3} J 16.4 \mathrm{~Hz}, 4 \mathrm{H}$, (vinyl) $\mathrm{H}^{1}$ ], $4.39(\mathrm{~m}, 8 \mathrm{H}), 2.15(\mathrm{~m}, 8 \mathrm{H}), 1.47(\mathrm{~m}, 24 \mathrm{H}), 1.37(\mathrm{~m}, 16 \mathrm{H}), 0.92(\mathrm{t}$, $\left.{ }^{3} J 6.8 \mathrm{~Hz}, 12 \mathrm{H}\right) ;{ }^{13} \mathrm{C}$ NMR [75 MHz, $\left.\left(\mathrm{CD}_{3}\right)_{2} \mathrm{CO}\right]: \delta$ 199.1, $198.7(1: 2$, $\mathrm{CO}), 152.4$ (pyridyl) $\mathrm{C}^{3}, 148.0$ (pyridyl) $\mathrm{C}^{1}, 137.3$ (phenyl) $\mathrm{C}^{1}, 136.0$ (vinyl) $\mathrm{C}^{2}, 128.5$ (phenyl) $\mathrm{C}^{2}, 125.9$ (vinyl) $\mathrm{C}^{1}, 123.2$ (pyridyl) $\mathrm{C}^{2}, 82.8$ $\left(\mathrm{CH}_{2}\right), 34.4\left(\mathrm{CH}_{2}\right), 32.6\left(\mathrm{CH}_{2}\right), 26.0\left(\mathrm{CH}_{2}\right), 23.3\left(\mathrm{CH}_{2}\right), 14.4\left(\mathrm{CH}_{3}\right)$; UV-VIS $\left(\mathrm{CH}_{3} \mathrm{CN}\right): \lambda_{\max }(\mathrm{nm}) 422$ (MLCT), 235, 285, 370 (LIG); emission: $\lambda_{\text {max }}$ (nm) 474, 490. Anal. for $\mathrm{C}_{84} \mathrm{H}_{100} \mathrm{~N}_{4} \mathrm{O}_{16} \mathrm{Re}_{4}$, found (calc.): C, 46.07 (46.57); H, 4.42 (4.65); N, 1.89 (2.59)\%. Mass (FAB, $\left.{ }^{187} \mathrm{Re}\right): \mathrm{m} / z$ $2168\left(\mathrm{M}^{+}\right)$. 
§ Crystallographic data: $\left[\mathrm{C}_{68} \mathrm{H}_{68} \mathrm{~N}_{4} \mathrm{O}_{16} \mathrm{Re}_{4}\right]$ 3a: $M=1942.06, T=150(1)$ $\mathrm{K}$, monoclinic, space group $=P 2_{1} / n, a=9.2304(1), \quad b=23.5898(1)$, $c=15.9339(2) \AA, \beta=98.796(1)^{\circ}, V=3428.69(6) \AA^{3}, Z=2, D_{\mathrm{c}}=1.881 \mathrm{~g}$ $\mathrm{cm}^{-3}, \mu=7.106 \mathrm{~mm}^{-1}, 24951$ reflections collected, 7796 independent reflections $\left(R_{\text {int }}=0.0420\right)$, refinement method: full-matrix least-squares on $F^{2}$. Final $R$ indices $[I>2 \sigma(I)]: R_{1}=0.0379, w R_{2}=0.0658, R$ indices (all data): $R_{1}=0.0512, w R_{2}=0.0699$, Largest diff. peak and hole: 1.187 and -0.786 e $\AA^{-3}$. CCDC reference numbers $156620-156622$. See http://www.rsc.org/suppdata/dt/b1/b101020o/ for crystallographic data in CIF or other electronic format.

1 S. Leininger, B. Olenyuk and P. J. Stang, Chem. Rev., 2000, 100, 853 and references therein; P. N. W. Baxter, J.-M. Lehn, G. Baum and D. Fenske, Chem. Eur. J., 1999, 5, 102; P. N. W. Baxter, J.-M. Lehn, B. O. Kneisel, G. Baum and D. Fenske, Chem. Eur. J., 1999, 5, 113; D. L. Caulder and K. N. Raymond, J. Chem. Soc., Dalton Trans., 1999, 1185 and references therein; D. L. Caulder and K. N. Raymond, Acc. Chem. Res., 1999, 32, 975; M. Fujita, Acc. Chem. Res., 1999, 32, 53; M. Fujita, Chem. Soc. Rev., 1998, 27, 417 and references therein; M. H. Keefe, K. D. Benkstein and J. T. Hupp, Coord. Chem. Rev, 2000, 205, 201; F. M. Dixon, A. D. Eisenberg, J. R. Farrell, C. A. Mirkin, L. M. Liable-Sands and A. L. Rheingold, Inorg. Chem., 2000, 39, 3432.

2 T. Imamura and K. Fukushima, Coord. Chem. Rev., 2000, 198 133; see, for example: Comprehensive Supramolecular Chemistry, eds. J. L. Atwood, J. E. D. Davies, D. D. MacNicol, F. Vogtle and J.-M. Lehn, Pergamon, Oxford, 1996, vols. 6 and 9; L. R. MacGillivray and J. L. Atwood, Angew. Chem., Int. Ed., 1999, 38, 1018; E. C. Constable and E. Schofield, Chem. Commun., 1998, 403.

3 S. Belanger, J. T. Hupp, C. L. Stern, R. V. Slone, D. F. Watson and T. G. Carrell, J. Am. Chem. Soc., 1999, 121, 557; R. V. Slone, K. D Benkstein, S. Belanger, J. T. Hupp, I. A. Guzei and A. L. Rheingold,
Coord. Chem. Rev., 1998, 171, 221; R. V. Slone, D. I. Yoon, R. M. Calhoun and J. T. Hupp, J. Am. Chem. Soc., 1995, 117, 11813; C. A. B. Brennan, D. I. Yoon, R. V. Slone, A. P. Kazala and J. T. Hupp, Inorg. Chem., 1996, 35, 2032.

4 S.-S. Sun and A. J. Lees, J. Am. Chem. Soc., 2000, 122, 8956.

5 K. D. Benkstein, J. T. Hupp and C. L. Stern, Angew. Chem., Int. Ed., 2000, 39, 2891; K. D. Benkstein, J. T. Hupp and C. L. Stern, J. Am. Chem. Soc., 1998, 120, 12982; K. D. Benkstein, J. T. Hupp and C. L. Stern, Inorg. Chem., 1998, 37, 5404.

6 S. M. Woessner, J. B. Helms, Y. Shen and B. P. Sullivan, Inorg. Chem., 1998, 37, 5406.

7 T. Rajendran, B. Manimaran, F.-Y. Lee, G.-H. Lee, S.-M. Peng, C. M. Wang and K.-L. Lu, Inorg. Chem., 2000, 39, 2016.

8 M. A. El-Sayed and H. D. Kaesz, Inorg. Chem., 1963, 2, 158.

9 C. Jiang, Y.-S. Wen, L.-K. Liu, T. S. A. Hor and Y. K. Yan, Organometallics, 1998, 17, 173.

10 P. J. Giordano and M. S. Wrighton, J. Am. Chem. Soc., 1979, 101, 2888; K. Kalyanasundaram, Photochemistry of Polypyridine and Porphyrin Complexes, Academic Press, London, 1992; T. J. Meyer, Pure Appl. Chem., 1986, 58, 1193.

11 R. V. Slone, J. T. Hupp, C. L. Stern and T. E. A. Schmitt, Inorg. Chem., 1996, 35, 4096.

12 L. A Worl, R. Duesing, P. Chen, L. D. Ciana and T. J. Meyer, J. Chem. Soc., Dalton Trans., 1991, 849.

13 R. Lin, Y. Fu, C. P. Brock and T. F. Guarr, Inorg. Chem., 1992, 31, 4346.

14 G. J. Stor, F. Hartl, J. W. M. van Outersterp and D. J. Stufkens, Organometallics, 1995, 14, 1115.

15 S. A. Moya, J. Guerrero, R. Pastene, R. Schmidt, R. Sariego, R. Sartori, J. S. Aparicio, I. Fonseca and M. M. Ripoll, Inorg. Chem., 1994, 33, 2341.

16 C. K. Johnson, ORTEP, Report ORNL-5138, Oak Ridge National Laboratory, Oak Ridge, TN, 1976. 